\title{
Optimization of Indoor Bluetooth Ranging Model Based on RSSI
}

\author{
Demei Peng, Liangfu Peng*, Yingying Yang \\ College of Electrical \& Information Engineering, Southwest Minzu University, Chengdu 610041, China \\ Email: 2011296353@qq.com
}

\begin{abstract}
Because the received signal strength indication (RSSI) ranging technology has problems with line-of-sight and multipath effects in indoor environments, the actual received RSSI value is unstable. In order to reduce the influence of RSSI value volatility on ranging accuracy, according to the fluctuation characteristics of the signal itself, a combined filtering method of Gaussian, median and mean is proposed to process the collected RSSI values, and the least squares method is used to fit and optimize the ranging parameter. Experiments show that using the RSSI intensity value processed by the combined filtering method to establish a model to achieve ranging, the maximum absolute error is about $2 \mathrm{~m}$, and the absolute average error is about $0.763 \mathrm{~m}$. The accuracy of the ranging has been significantly improved, and the ranging model has been optimized.
\end{abstract}

Keywords: RSSI, ranging model, least square method, combined filtering

\section{Introduction}

In recent years, with the continuous improvement of urban intelligence, people have more and more demand for location-based services. Indoor positioning technology has become an important research direction. Providing people with high-precision positioning is a current research hotspot. Indoor positioning technology mainly includes Infrared Technique, Radio Frequency Identification, Ultra Wide Band, WIFI, Bluetooth, etc. Among these positioning methods, the positioning technology based on iBeacon has become a research hotspot in indoor positioning due to its low power consumption, low cost, and easy implementation.

At present, most indoor positioning is based on received signal strength indication ranging [1], and ranging is an important part of indoor positioning. Due to the complex and changeable indoor environment, the literature [2] analysis pointed out that the propagation channel of wireless signals is susceptible to interference from various noises in the environment, resulting in the received signal strength indication (RSSI) at the same location around a certain central value Fluctuation, that is, the RSSI value obeys the Gaussian distribution, and direct use will cause large errors. In response to this problem, the literature [3] used data and graphs to quantitatively analyze the size of RSSI influencing factors in terms of power, relative angles of receiving and sending points, multi-node receiving and sending, and environmental factors. At the same time, the logarithmic attenuation model of RSSI was obtained by analyzing the measured data, but because it did not do any processing on the RSSI value, the influence of the fluctuation of the data on the final positioning distance was ignored. Literature [4] uses unidirectional outlier removal and moving average to preprocess the RSSI, and then calculates the distance and uses Kalman filter to filter. The experimental results show that the Kalman filter algorithm is used to process the data after removing the outliers, so that the average positioning accuracy is significantly improved, but the environmental factors in the ranging model are not measured in combination with the actual environment. Finally, the influence of the increase of distance on the accuracy of ranging was not considered when modeling. Literature [5] establishes a regional discriminant model for RSSI signals and constructs RSSI vector groups for different regions, uses Bayesian estimation analysis to calculate weighted values for RSSI intensity ranging, determines the precise region when RSSI is received, and selects the RSSI vector of this region Multi-point centroid positioning is performed, but the algorithm is more complicated and computationally expensive, and its practical application value is not great. Reference [6] aimed at the problems of non-line-of-sight and multipath transmission in the indoor environment based on the received signal strength indicator ranging technology, and the relatively large ranging error, a multiple filtering algorithm was used to filter the collected RSSI values. The least squares method is used to fit the parameters of the signal attenuation model to obtain parameter values that meet 
the specific environment. However, in the actual environment, after the Bluetooth signal propagates over a certain distance, the distance corresponding to a small decrease in the RSSI value will increase a lot. Therefore, after the distance increases, the accuracy of the model using the least squares fitting will also decrease, and the signal needs to be determined. Target optimal transmission distance for positioning optimization.

In view of the shortcomings of the above-mentioned research, this paper analyzes and deals with the instability and range resolution of RSSI in the indoor Bluetooth ranging model. First, according to the fluctuation characteristics of the RSSI value, Gaussian filtering is used to eliminate the larger values, and then median filtering is performed on the processed data. Finally, the average value after filtering is taken as the sample value and the least square method is used to establish a Bluetooth ranging model. Secondly, considering that after the Bluetooth signal propagates over a certain distance, the distance corresponding to a small decrease in the RSSI value will increase a lot, so the fitting accuracy of the model will also decrease. In order to improve the accuracy of the ranging model, this article chooses to measure through experiments. The best transmission fitting distance from the model to further improve the accuracy of the model.

\section{RSSI Signal Data Processing Method}

Like general wireless signals, RSSI signals are susceptible to many factors. In the actual ranging, as shown in Figure 1, the RSSI signal is very unstable when affected by factors such as multipath and non-line-of-sight, and has great volatility, which causes it to deviate from the true value.

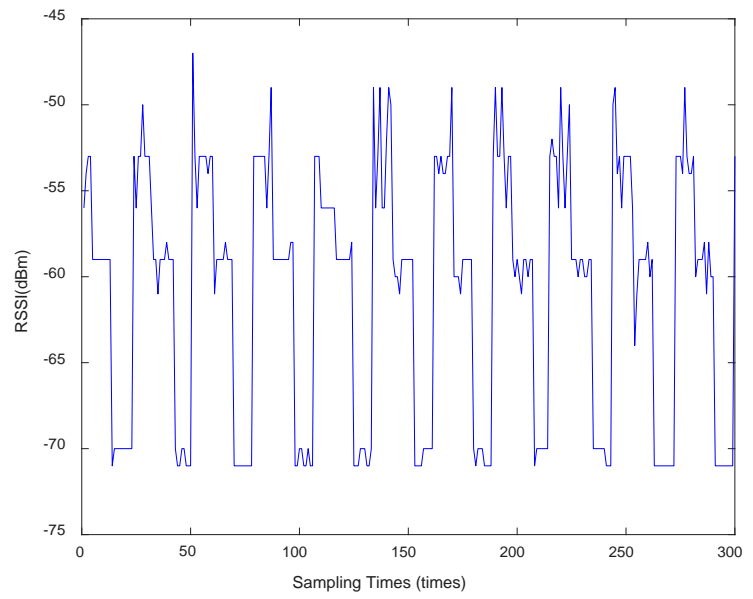

Figure 1. Fluctuation diagram of RSSI signal received at a distance of $1 \mathrm{~m}$

According to the literature, the instability of the RSSI signal can be reduced by certain filtering algorithms, thereby improving the ranging accuracy. The following will briefly introduce the commonly used mean and median filter models, and on this basis, propose the combined filter model used in this article.

\subsection{Mean Filter Model}

Mean filtering means that the node calculates the arithmetic average of a set of RSSI values received, as shown in equation (1), and the average is used as the measurement result. When the number of samples $n$ is large or the RSSI signal fluctuation is very small, the problem of randomness of RSSI data can be better solved, but when the number of samples is small or the RSSI value fluctuates greatly, the credibility of this method will decrease [7].

$$
\overline{R S S I}=\frac{1}{n} \sum_{i=1}^{n} R S S I_{i}
$$




\subsection{Median Filtering Model}

Median filtering is a non-linear data filtering method. In the case of one-dimensional data, the median filter is a window containing an odd number of data. After processing, the median value of the window is output as the final result. Suppose the signal strengths received at the distance from the transmitting node $d$ are $\mathrm{RSSI}_{1}, \mathrm{RSSI}_{2}, \mathrm{RSSI}_{3}, \mathrm{RSSI}_{n}$, and $n$ is the length of the filter window, and perform median filtering on this sequence. Arrange the intensity values in the sequence according to the magnitude of the value, and then take the intermediate value as the filtered result and output, as shown in equation (2). The median filter eliminates the influence of outliers on the final measurement result to a large extent, but when the number of samples is small or the RSSI value fluctuates greatly, the filtering result of the median filter method is not very effective.

$$
R_{d}=\operatorname{Med}\left\{\mathrm{RSSI}_{1}, \mathrm{RSSI}_{2}, \ldots \mathrm{RSSI}_{n}\right\}
$$

\subsection{Combined Filter}

A large number of experimental studies have shown that the RSSI random distribution is approximately in line with the Gaussian distribution model. The collected RSSI signals are sorted, and the signal at $1 \mathrm{~m}$ is taken as an example, and the probability distribution diagram shown in Figure 2 is obtained by statistics. It can be seen from Figure 2 that the Gaussian model can better fit the distribution of RSSI in the actual environment.

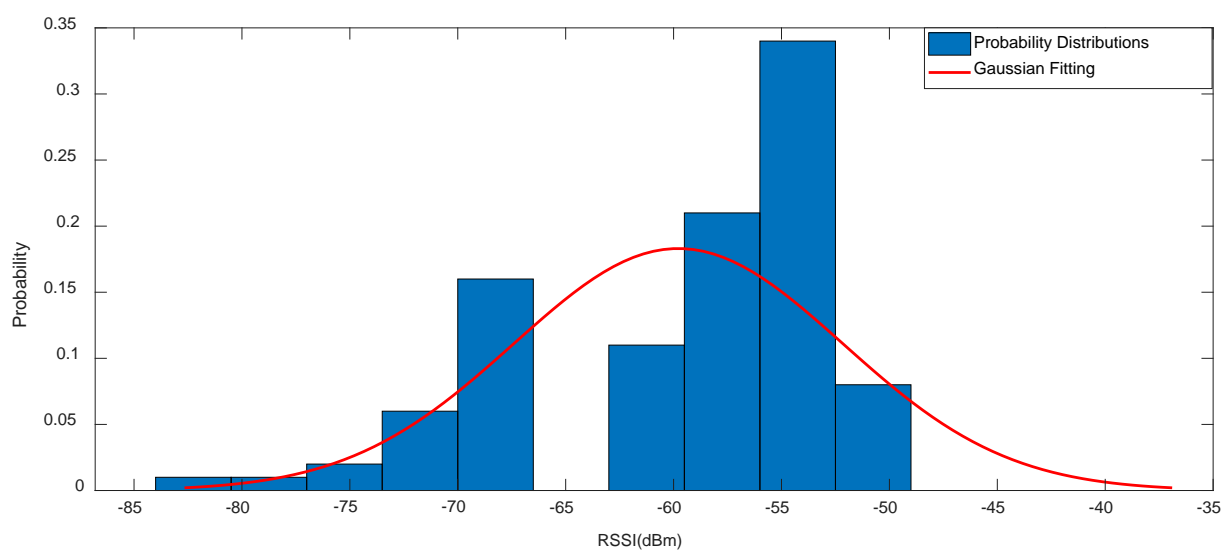

Figure 2. Gaussian distribution of RSSI value at $1 \mathrm{~m}$

The probability density function of the Gaussian model [8, 9] is as follows.

$$
\begin{gathered}
F(R S S I)=\frac{1}{\sigma \sqrt{2 \pi}} \cdot e^{-\frac{(R S S I-\mu)^{2}}{2 \sigma^{2}}} \\
\mu=\frac{1}{n} \sum_{i=1}^{n} R S S I_{i} \\
\sigma^{2}=\frac{1}{n-1} \sum_{i=1}^{n}\left(R S S I_{i}-\mu\right)
\end{gathered}
$$

In the formula, $\operatorname{RSSI}_{i}$ is the $i$-th RSSI intensity value; $n$ is the number of collections; $\mu$ is the mean value in the normal distribution; $\sigma$ represents the degree of data dispersion.

Under the normal curve, the area of a certain interval on the horizontal axis directly reflects the probability that the variable value falls within the interval. Through data analysis, select the data in the high probability interval $\left(\mu-\sigma \leqslant \mathrm{RSSI}_{i} \leqslant \mu+\sigma\right)$, the probability of occurrence is 0.6826 , take out all the RSSI measurement values in this range, and find the average value as Gaussian filter The output of the device. However, only using Gaussian filtering cannot completely eliminate data fluctuations. Therefore, only using Gaussian filtering in RSSI signal preprocessing does not have a good effect. In order 
to achieve a smooth and stable output of the RSSI sample data, it is necessary to continue processing the sample data.

The specific implementation process of the combined filtering proposed in this paper is: first establish a Gaussian model, select high probability occurrence values from the collected RSSI measurement values, then perform median filtering on the selected data, and finally take the filtered RSSI value The average is used as the final RSSI sample value.

\section{Bluetooth Ranging Model}

Studies have shown that during the propagation of wireless signals, the signal strength will gradually weaken as the distance increases. Therefore, this paper uses a logarithmic distance attenuation model for the received RSSI signal strength, and the transmission model is as shown in formula (6).

$$
P(d)=P\left(d_{0}\right)-10 n \lg \left(\frac{d}{d_{0}}\right)+\varepsilon
$$

In formula $(6), d_{0}$ is the reference distance; $d$ is the distance between the transmitter and the receiver; $P\left(d_{0}\right)$ represents the signal strength received by the receiver when the distance is $d_{0} ; P(d)$ represents the signal received by the receiver when the distance is $d ; n$ is the path loss index, which is closely related to the surrounding environmental factors, usually obtained from actual measurement; $\varepsilon$ is caused by the fading effect and obeys a random variable with a mean value of zero and a variance of a Gaussian normal distribution of $\sigma^{2} ; P\left(d_{0}\right), P(d), \varepsilon$ are all in $\mathrm{dBm}$.

The actual received signal strength $P(d)$ can be expressed by the RSSI value. The logarithmic distance path loss model formula in equation (7) is converted into the corresponding RSSI value form. The attenuation model formula is as follows.

$$
R S S I=A-10 n \lg \left(\frac{d}{d_{0}}\right)+\varepsilon
$$

In the formula, RSSI $=P(d) ; A=P\left(d_{0}\right)$; RSSI and $A$ are the RSSI strength values received at the distance between the receiving end and the transmitting end of $d$ and $d_{0}$, respectively.

\section{Model Parameter Optimization}

\subsection{Establish a Path Loss Model Based on the Least Square Method}

In order to allow the RSSI ranging model to more truly reflect the propagation characteristics of the actual indoor environment and ensure the accuracy of the ranging, the constant $A$ and the path loss index $n$ need to be optimized to obtain parameter values that conform to the indoor environment. The value of constant $A$ and path loss index $n$ is estimated by the principle of least squares, and the data corresponding to $\overline{R S S I}_{d i}$ and distance di is obtained according to the RSSI measurement value after the filtering process, as shown in equation (8).

$$
\begin{gathered}
\overline{R S S I}_{d i}=A-10 n \lg d i \\
\mathbf{K X}=\mathbf{B}
\end{gathered}
$$

In the formula,

$$
\mathbf{K}=\left[\begin{array}{cc}
1 & -10 \lg d_{1} \\
1 & -10 \lg d_{2} \\
\vdots & \vdots \\
1 & -10 \lg d_{i}
\end{array}\right] ; \quad \mathbf{B}=\left[\begin{array}{c}
{\overline{R S S I_{d 1}}}_{\overline{R S S I}_{d 2}} \\
\vdots \\
\overline{R S S I}_{d i}
\end{array}\right] ; \quad \mathbf{X}=\left[\begin{array}{c}
A \\
n
\end{array}\right]
$$

According to the principle of least square method, the estimated value of parameter $X$ can be obtained.

$$
\mathbf{X}=\left(\mathbf{K}^{T} \mathbf{K}\right)^{-1} \mathbf{K}^{T} \mathbf{B}
$$

The estimated values of model parameters, can be obtained by formula (10), and the equation of the RSSI ranging model can be obtained by the least squares curve fitting method [10, 11]. 


\subsection{Best Transmission Fitting Distance}

In order to improve the accuracy of the experiment, the method in [12] is used to test the best transmission fitting distance of the beacon. The measured RSSI value of $1 \sim 8 \mathrm{~m}$ is shown in Figure 3.

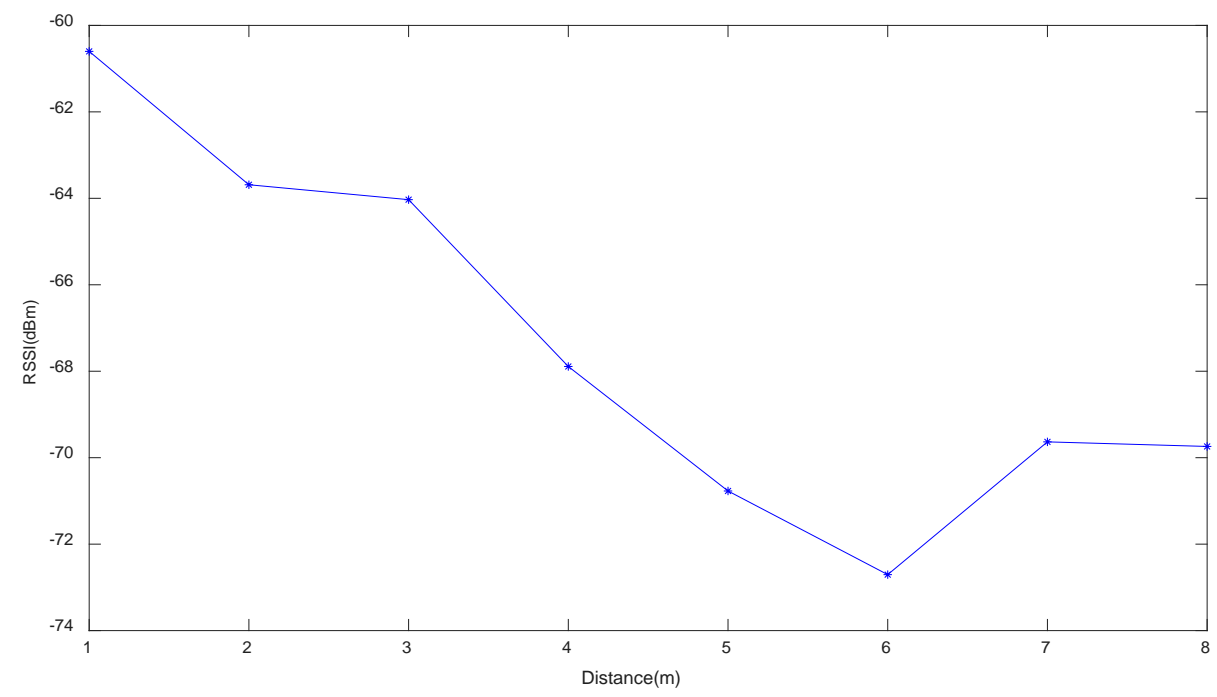

Figure 3. RSSI change value at $1 \sim 8 \mathrm{~m}$

It can be seen from Figure 3 that the Bluetooth signal is affected by the indoor complex environment. When the Bluetooth beacon is farther away from the receiving point, the signal fluctuates more, especially the data after 6 meters has a very obvious jump phenomenon. Therefore, filtering processing and determining the best transmission distance are essential for data modeling.

\subsection{Experimental Description and Results}

The experiment uses the iBeacon beacon whose main chip is TI CC2640R2F as the signal transmitter. During the experiment, the transmit power of the beacon was set to $0 \mathrm{dBm}$, and the broadcast interval was set to 100ms. The receiving end is the NRF52832 collection module, which can complete the RSSI collection of iBeacon equipment.

In order to reduce the influence of the wall and the ground on the signal strength, using the aisle environment of the laboratory, place the iBeacon beacon on a tripod $1.2 \mathrm{~m}$ above the ground, and keep the beacon and the signal collector on the same horizontal line. During the data collection process, by changing the position of the beacon or signal collector so that the distance between the signal collector and the beacon is $0.25 \mathrm{~m}, 0.5 \mathrm{~m}, 0.75 \mathrm{~m} \ldots .5 \mathrm{~m}$, and 5000 sets of data are collected at each distance node position. By drawing the RSSI signal fluctuation graph, analyzing its change trend, choosing more stable 1000 sets of data, using improved combined filtering to process these data, and combining the above least square method to fit the filtered RSSI value and distance. The result is shown in Figure 4, the optimized parameter $A=-59.98, n=0.9875$. 


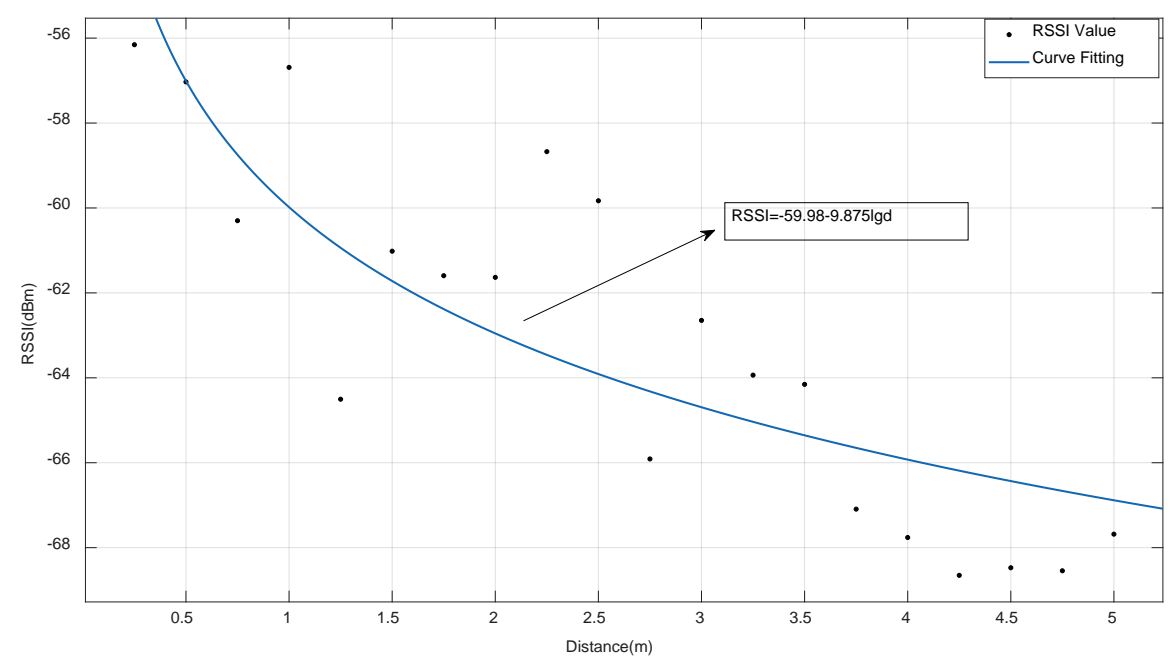

Figure 4. Fitting curve diagram after combined filtering within $1 \sim 5 \mathrm{~m}$

\subsection{Error Analysis}

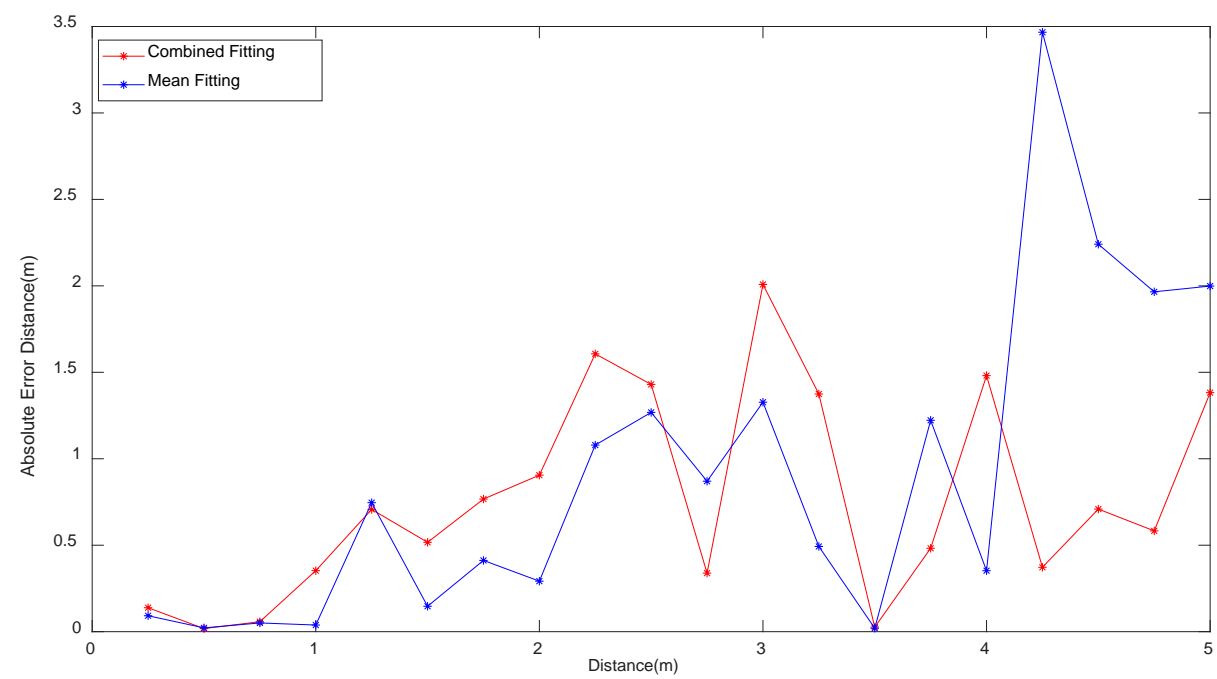

Figure 5. Error curve of ranging

Using mean filtering and combined filtering to model, and verifying the distance of 100 sets of data at each distance node, the absolute distance measurement error value is shown in Figure 5. It can be seen from Figure 5 that the maximum absolute error after mean filtering is $3.467 \mathrm{~m}$, and the absolute average error is $0.905 \mathrm{~m}$; the maximum absolute error after combined filtering is $2.008 \mathrm{~m}$, and the absolute average error is $0.763 \mathrm{~m}$. It can be seen that the overall error of the combined filtering is reduced, and after $3.5 \mathrm{~m}$, the ranging accuracy is greatly improved.

\section{Conclusion}

In this paper, the Bluetooth-based RSSI ranging technology uses a combination of Gaussian filtering, median and average filtering to process data, and filters the collected Bluetooth signal strength values. According to the actual environment of the experiment, the best transmission fitting distance is determined, and a distance measurement model based on the least square method is established, which improves the fitting accuracy of the distance measurement model. Experimental results show that the 
improved ranging method proposed in this paper can accurately and effectively output signal strength values, reduce ranging errors, improve ranging accuracy based on Bluetooth RSSI, and lay a foundation for subsequent indoor positioning work.

Acknowledgements. The work of this paper is supported by the Southwest Minzu University Graduate Innovative Research Project (Master Program CX2020SZ94). A special acknowledgement should give to Southwest Minzu University for its experimental conditions and technical support.

\section{References}

1. Yan K, Zhou H, Xiao H, et al. Current status of indoor positioning system based on visible light[C]// 2015 15th International Conference on Control, Automation and Systems (ICCAS). IEEE, 2015.

2. Zhang Jianwu, Zhang Lu, Ying Ying, et al. Research on RSSI ranging based on ZigBee[J]. Journal of Sensor Technology, 22, 2 (2009), 285-288.

3. Li Zaiyu. Research and realization of RSSI positioning principle. Radio Engineering[J], 43, 07 (2013), 8-10.

4. Song Chai,Renbo An,Zhengzhong Du. An Indoor Positioning Algorithm Using Bluetooth Low Energy RSSI[A]. Science and Engineering Research Center.Proceedings of 2016 International Conference on Advanced Materials Science and Environmental Engineering (AMSEE2016)[C].Science and Engineering Research Center:Science and Engineering Research Center,2016:3.

5. Peng Hao, Peng Min, An Ning, et al. Bayesian area discrimination positioning algorithm based on Bluetooth RSSI[J]. Computer Engineering, 45, 03 (2019), 125-137.

6. Tao Jiafeng, Yang Xiaohong, Wang Jianping, et al. Indoor ranging model optimization technology based on RSSI[J]. Sensors and Microsystems, 36, 1 (2017), 24-26,30.

7. Tao Weige, Zhu Yihua, Jia Ziyan. Ranging algorithm based on RSSI hybrid filtering and least square parameter estimation[J]. Journal of Sensor Technology, 25, 12 (2012), 1748-1753.

8. Liang Min, Ma Kai. Research on echo signal denoising method based on Gaussian filtering. Surveying and Spatial Geographic Information[J], 40, 01 (2017), 40-42.

9. Bi Jingxue, Wang Yunjia, Ning Yipeng, et al. Indoor ranging and positioning method considering BLE beacon geometry optimization[J]. Journal of China University of Mining and Technology, 50, 02 (2021), 411-416.

10. Zhu Minghui, Zhang Huiqing. Research on the indoor ranging model based on RSSI[J]. Sensors and Microsystems, 29, 08 (2010), 19-22.

11. Yue Junmei, Peng Xinguang, Li Qingyi, et al. Research on underground coal mine positioning based on RSSI ranging $[\mathrm{J}]$. Coal Engineering, 50, 08 (2018), 122-125.

12. Zhang Yalei, Wang Jian, Han Houzeng, et al. Research and accuracy analysis of Bluetooth indoor ranging model[J]. Science of Surveying and Mapping (2020), 1-11. 